\title{
THE VISUAL CONTROL OF BICYCLE STEERING: THE EFFECTS OF SPEED AND PATH WIDTH
}

\author{
Pieter Vansteenkiste, Greet Cardon, Eva D'Hondt, Renaat Philippaerts, Matthieu Lenoir \\ Department of Movement and Sports Sciences, Ghent University, Belgium, \\ pieter.vansteenkiste@ugent.be, greet.cardon@ugent.be, eva.dhondt@ugent.be, renaat.philippaerts@ugent.be, \\ matthieu.lenoir@ugent.be \\ Corresponding author: pieter.vansteenkiste@ugent.be
}

Ghent University, Department of Movement and Sports Sciences

Watersportlaan 2, 9000 Ghent, Belgium

Tel: +32 (0) 9.264.86.83

E-mail: pieter.vansteenkiste@ugent.be

Article published in:

Accident Analysis and Prevention; Volume 51, March 2013, Pages 222-227

doi: 10.1016/j.aap.2012.11.025

\begin{abstract}
Although cycling is a widespread form of transportation, little is known about the visual behaviour of bicycle users. This study examined whether the visual behaviour of cyclists can be explained by the twolevel model of steering described for car driving, and how it is influenced by cycling speed and lane width. In addition, this study investigated whether travel fixations, described during walking, can also be found during a cycling task. Twelve adult participants were asked to cycle three fifteen meter long cycling lanes of 10, 25 and $40 \mathrm{~cm}$ wide at three different self-selected speeds (i.e., slow, preferred and fast). Participants' gaze behaviour was recorded at $50 \mathrm{~Hz}$ using a head mounted eye tracker and the resulting scene video with overlay gaze cursor was analyzed frame by frame. Four types of fixations were distinguished: (1) travel fixations, (2) fixations inside the cycling lane (path), (3) fixations to the final meter of the lane (goal), and (4) fixations outside of the cycling lane (external). Participants were found to mainly watch the path $(41 \%)$ and goal $(40 \%)$ region while very few travel fixations were made $(<5 \%)$. Instead of travel fixations, an optokinetic nystagmus was revealed when looking at the near path. Large variability between subjects in fixation location suggests that different strategies were used. Wider lanes resulted in a shift of gaze towards the end of the lane and to external regions, whereas higher cycling speeds resulted in a more distant gaze behaviour and more travel fixations. To conclude, the two-level model of steering as described for car driving is not fully in line with our findings during cycling, but the assumption that both near and far regions are necessary for efficient steering seems valid. A new model for visual behaviour during goal directed locomotion is presented.
\end{abstract}

\section{Keywords}

Two-level model; travel fixation; optokinetic nystagmus; cycling; lane keeping; gaze constraints 


\section{Introduction}

Goal directed locomotion is usually guided by information from task specific visual search patterns (Ballard and Hayhoe, 2010). Both in car driving and walking, eye movements have been studied to understand how humans use vision for obstacle avoidance and safe navigation (Marigold et al., 2007; Patla and Greig, 2006; Hildreth et al., 2000; Mourant and Rockwell, 1972; Underwood et al., 2003; Falkmer and Gregersen, 2005). Unfortunately, despite the fact that cycling is a widespread form of transportation and is often recommended as a healthy and economic way to do so (Rabl and de Nazelle, 2012), little is known about the visual behaviour of cyclists. With increased use of bicycles for transportation, the number of bicycle accidents also increased (Juhra et al., 2011). Therefore, greater insight in the visual behaviour of cyclists is essential for effective traffic education and infrastructural planning.

In car driving, gaze behaviour can be best described by the two-level model of steering (Donges, 1978; Land and Horwood, 1995). According to this model, two visual regions are used for efficient steering. First, a distant point on the travel path is used for controlling heading. On a straight road this is usually the vanishing point, a leading car or a point to which the car has to be steered (Salvucci and Gray, 2004; Land and Lee, 1994). On curved roads, the 'tangent point' has been identified as an important visual cue. This is the point on the inside edge of the bend which protrudes the most in the road and has been shown to be closely linked to the drivers steering behaviour (Land and Lee, 1994; Mars, 2008; Kandil et al., 2009). The second region described in the two-level model is the near road region. This region includes the road and its markings in the immediate proximity of the car and plays an important role for lane keeping. However, this near region is rarely fixated. Instead of switching gaze from far road to near road regions, car drivers tend to fixate mainly the far road and attend to the near road peripherally for position-in-lane feedback. This gaze strategy has been shown to be efficient for multiple steering tasks, such as corrective steering, lane changing and curve negotiation (Salvucci and Gray, 2004).
The two-level model has not yet been explicitly tested for walking but some studies in real-world scene perception also use the distinction between near and far regions (Foulsham et al., 2011; Pelz and Rothkopf, 2007). In contrast to car driving, the near path region is frequently gazed at during walking $( \pm 30 \%$ of the time) and only few fixations $(<10 \%)$ are made to the distant path (Foulsham et al., 2011). On an uneven pathway, proportion of gaze to the near path even increases up to 75\% (Pelz and Rothkopf, 2007). This 'path-watching' phenomenon can be explained by pedestrians being more dependent on path quality for maintaining dynamic balance, in contrast to car drivers. The fact that pedestrians also spend more time looking at regions irrelevant for the control of locomotion, such as the scenery (Turano et al., 2003), is probably due to the lower speeds at which they travel. At lower speeds, more time is available to anticipate and react to possible hazards, which leads to a lower task demand.

Patla and Vickers (2003) mentioned a specific type of near path fixation during walking, called the travel fixation. A travel fixation occurs when gaze is held on a fixed distance about two steps in front of the body and is carried along at the speed of locomotion. As a result, the point of regard moves at the same speed as the body. During this type of fixation optic flow lines pass through central as well as peripheral vision and are used to obtain information about the self-motion. However, generalization of travel fixations to other forms of locomotion can be questioned since only few other studies described their use (Fowler and Sherk, 2003; Hollands et al., 2002). In addition, the use of travel fixations would mean that the OptoKinetic Reflex (OKR) is suppressed. This OKR is a reflexive eye movement that stabilizes the retinal image by adapting the eye movement velocity to that of the retinal image (Lappe and Hoffman, 2000; Miles, 1998). When moving forward at a constant speed, the environment is perceived as a constant radial optic flow. Without eye movements adapted to the speed of the optic flow, visual pick-up would be blurry. Therefore, a series of optokinetic eye movements is elicited (Solomon and Cohen, 1992; Lappe et al., 1998; Knapp et al., 2008; Niemann et al., 1999). This series of OKR's is called OptoKinetic Nystagmus 
(OKN) and has been described during simulated rectilinear self motion in the monkey and humans (Lappe et al., 1998; Niemann et al., 1999) and recently also during car driving (Authié and Mestre, 2011). To our knowledge, however, neither travel fixations nor OKN have been described for cycling.

Visual behaviour for walking and for car driving is different, but in both cases humans seem to rely on a synergy of far and near road information. The first aim of this study is to examine whether the visual behaviour of cyclists can be explained by the two-level model of steering. If this model can be applied to cycling as for driving, cyclists would mainly look at distant points while they maintain centred in the lane by attending the proximal pathway peripherally. However, Land and Horwood ( 1995) noted that at lower speeds $(<12 \mathrm{~m} / \mathrm{s})$ the near-road information is adequate on its own. In addition, Pelz and Rothkopf (2007) showed that when task demands were higher, vision shifted towards the near region. Therefore, the second aim of this experiment was to test the influence of these constraints on gaze behaviour by imposing three cycling speeds and three lane widths (i.e., a smaller path). It was expected that higher cycling speeds and lower task demands would result in a more distant visual behaviour. Finally, the third aim of this study was to investigate whether travel fixations are made or an $\mathrm{OKN}$ is elicited when looking at the path during a in-situ linear cycling task.

\section{Material and methods}

\subsection{Subjects}

A convenience sample of nineteen participants took part in the experiment and were recruited from Ghent University's students and staff. Twelve participants aged
21 to 28 (five females) who's tracking ratio of eye movements (i.e., the time that direction of gaze could be determined / duration of trial) was at least $85 \%$ and for whom pre and post calibration were good, were selected for further analysis. All participants had normal or corrected-to-normal vision and all used their bicycle on regular basis for transportation.

\subsection{Apparatus}

Eye movements were recorded using the IviewX Head mounted Eye tracking Device (SMI, Teltow GER). An infra red eye camera was mounted on a baseball cap and recorded the left eye movements at $50 \mathrm{~Hz}$ using pupil position and corneal reflex. A scene camera with $3.6 \mathrm{~mm}$ lens, placed next to the eye camera recorded video images at $25 \mathrm{~Hz}$ with a horizontal and vertical field of view of approximately $33^{\circ}$. Both cameras were connected to a notebook which was worn in a backpack. Video and eye tracking data were combined using SMI's software BeGaze 3.0. The system has a spatial accuracy of $1^{\circ}$.

\subsection{Set up and procedure}

The experimental set up is shown in Figure 1. Cycling lanes of $10 \mathrm{~cm}$ (Narrow), $25 \mathrm{~cm}$ (Middle) and $40 \mathrm{~cm}$ (Wide) of width and $15 \mathrm{~m}$ of length were marked on the floor with a white tape. Two mechanical gates at the start and at the end of the lane gave a visual signal when the cyclist passed through. A line marked the start of the $15 \mathrm{~m}$ run-up before the first gate and an overview camera $(25 \mathrm{~Hz}$, Full HD) stood four meters behind the second gate to record the cyclist and the signals of the mechanical gates. After calibration, participants were given one familiarization trial for each lane. Subsequently, the test leader (A on Fig.1) asked the participant to cycle through one of the lanes at self-
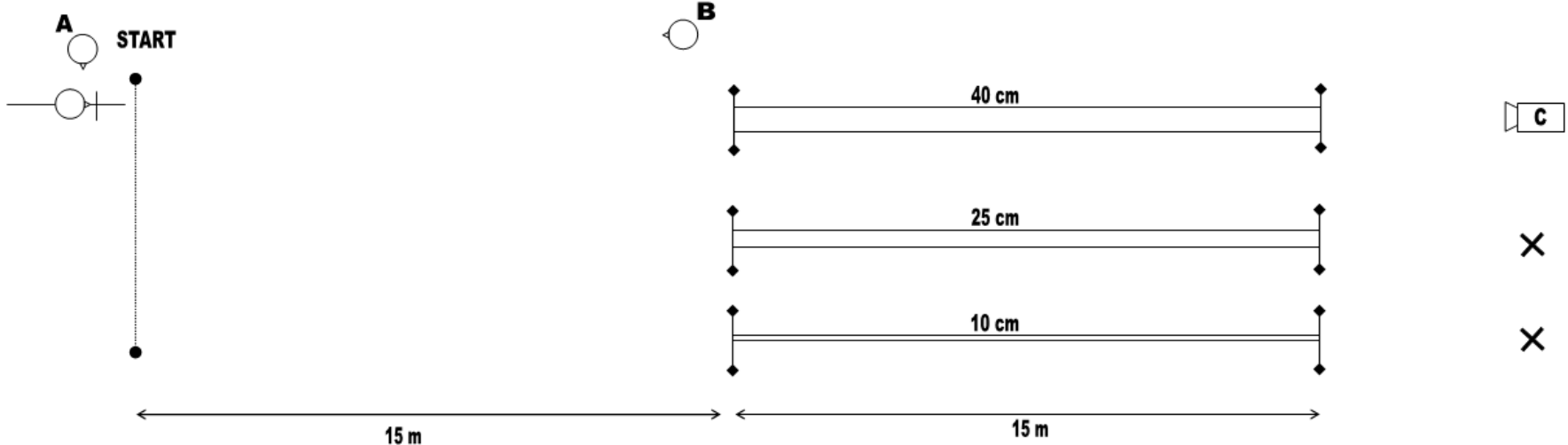

Figure 1 : Experimental set-up. (A) test leader that gives instructions to the participant; (B) test leader with clapperboard; $(C)$ and $(X)$ places of overview camera 
selected low, preferred or high speed without crossing the edge lines. A start signal was given to the cyclist by a second test leader (B on Fig.1) using a clapperboard. This signal was also used for synchronizing eye tracking data with the video images of the overview camera. Nine conditions (3 lanes x 3 speeds) were carried out in a randomized order. After the last condition a calibration check was done. All tests were done in a gymnasium with a parquet floor and with a standard city bicycle (women's model) rented from the university bicycle service. Saddle height was adjusted for each participant so that they could reach the ground with the tips of their feet while being seated on it.

\subsection{Data analysis :}

Performance variables were cycling speed $(\mathrm{v}=$ ( $15 \mathrm{~m} /$ cycling time from gate 1 to gate 2 in $\left.\mathrm{sec})^{*} 3,6\right)$ expressed in $\mathrm{km} / \mathrm{h}$ and the cycling time inside the edge lines, expressed as a percentage of total cycling time (inlane\%). The in-lane cycling time was visually obtained from the overview camera at the end of the lane. For each trial the video data with overlay gaze cursor were exported from BeGaze 3.0. These videos were analyzed frame by frame in Kinovea to measure fixation duration, fixation type, and fixation location. A fixation was defined as the cursor being steady for at least three consecutive frames (120ms) and ended when a saccade was made. This "direct inspection" method has been reported to be time-consuming but very effective for head mounted eye trackers (Duchowski, 2007, p138). Single Measure Intraclass Correlation was calculated on $8.33 \%$ of the data to test the intrarater reliability. Results demonstrated a high intrarater reliability for both the number of fixations $(\mathrm{R}=0.856)$ and the fixation duration $(\mathrm{R}=0.902)$. Travel fixations were denoted when "gaze was held stable in front on the travel surface and moved at the speed of locomotion" (Patla and Vickers, 1997). All other fixations were divided into fixations on the cycling path (Path), on the final meter of the cycling lane (Goal) and on areas outside of the travel path (External). A total fixation percentage (TotFix\%) was calculated by dividing the total fixation duration of a trial by the duration of that trial. Fixation time of each fixation type (Fix\%) was expressed as a percentage of the total fixation time, (\%Travel, \%Path, \%Goal and \%External).

\subsection{Statistics}

Effects of self-imposed cycling speed and lane width on actual cycling speed, in-lane\% and TotFix\% were analyzed using a repeated measures ANOVA, including two within subject factors with each of them consisting of three sublevels (i.e., Speed: Slow, Preferred, Fast; Width: Narrow, Middle and Wide). For analysis of the effect of speed and width on Fix\%, a similar repeated measures ANOVA was used but the four types of fixations were added as separate measures. For comparison of the Fix\% of the four types of fixation, a repeated measures ANOVA was done with three within subject factors (width, speed and fixation type). Significant differences $(\mathrm{p}<0.05)$ were further analyzed using pairwise comparisons.

\section{Results}

Table 1 provides an overview of both descriptive and comparative statistics.

\subsection{Cycling Speed, in-lane\% and total fixation\%}

Cycling speed increased from the Slow to the Fast condition $(\mathrm{p}<0.001)$ and participants cycled faster on the Wide lane as compared to the Narrow lane $(\mathrm{p}<0.045)$. Cycling speed on the Middle lane appeared to be lower than on the Wide lane and higher than on Narrow lane but these differences did not reach significance $(\mathrm{p}=0.070$ and $\mathrm{p}=0.085$, respectively). In-lane $\%$ was significantly lower on the Narrow lane than on the Middle $(\mathrm{p}<0.001)$ and on the Wide lane $(\mathrm{p}<0.001)$. No difference between Middle and Wide lane speeds were found $(\mathrm{p}=0.060)$. Cycling speed did not influence the in-lane\%. TotFix\% at Low speed $(74 \pm 11 \%)$ was significantly higher than in Preferred speed $(68 \pm 13 \% ; \mathrm{p}=0.003)$ and High speed (66 $\pm 17 \% ; \mathrm{p}=0.014)$. No interaction between speed and width and no effect of lane width was found on TotFix\%. 


\begin{tabular}{|c|c|c|c|c|c|c|c|c|c|c|c|c|}
\hline & \multicolumn{3}{|c|}{ Narrow } & \multicolumn{3}{|c|}{ Middle } & \multicolumn{3}{|c|}{ Wide } & \multicolumn{3}{|c|}{$F$-value (df) and $p$} \\
\hline & Slow & Preferred & Fast & Slow & Preferred & Fast & Slow & Preferred & Fast & Width & Speed & $W * P$ \\
\hline \multicolumn{13}{|c|}{ speed $(\mathrm{km} / \mathrm{h})$} \\
\hline Mean & 8,48 & 11,97 & 16,76 & 8,66 & 12,19 & 17,08 & 8,81 & 12,50 & 17,76 & $4.607(22)$ & $63.225(22)$ & $0.544(44)$ \\
\hline $\mathrm{SD}$ & 2,22 & 2,04 & 2,95 & 2,25 & 1,86 & 2,97 & 2,27 & 2,03 & 3,62 & 0.021 & $<0.001$ & 0.704 \\
\hline \multicolumn{13}{|c|}{ in-lane\% } \\
\hline Mean & $57 \%$ & $65 \%$ & $61 \%$ & $98 \%$ & $99 \%$ & $100 \%$ & $100 \%$ & $100 \%$ & $100 \%$ & $53.256(22)$ & $0.639(22)$ & $0.582(44)$ \\
\hline $\mathrm{SD}$ & $20 \%$ & $22 \%$ & $26 \%$ & $5 \%$ & $3 \%$ & $1 \%$ & $0 \%$ & $0 \%$ & $0 \%$ & $<0.001$ & 0.512 & 0.556 \\
\hline \multicolumn{13}{|c|}{ total fix\% } \\
\hline Mean & $73 \%$ & $67 \%$ & $69 \%$ & $77 \%$ & $72 \%$ & $70 \%$ & $71 \%$ & $66 \%$ & $60 \%$ & $2.174(10)$ & $9.457(10)$ & $0.182(8)$ \\
\hline SD & $12 \%$ & $14 \%$ & $17 \%$ & $4 \%$ & $9 \%$ & $15 \%$ & $13 \%$ & $17 \%$ & $20 \%$ & 0.165 & 0.005 & 0.941 \\
\hline \multicolumn{13}{|c|}{$\%$ Path fix } \\
\hline Mean & $69 \%$ & $50 \%$ & $47 \%$ & $51 \%$ & $36 \%$ & $27 \%$ & $31 \%$ & $31 \%$ & $25 \%$ & $19.670(22)$ & $5.172(22)$ & $1.239(44)$ \\
\hline $\mathrm{SD}$ & $17 \%$ & $25 \%$ & $27 \%$ & $32 \%$ & $25 \%$ & $29 \%$ & $32 \%$ & $29 \%$ & $31 \%$ & $<0.001$ & 0.014 & 0.308 \\
\hline \multicolumn{13}{|c|}{$\%$ Travel fix } \\
\hline Mean & $1 \%$ & $4 \%$ & $4 \%$ & $1 \%$ & $2 \%$ & $5 \%$ & $1 \%$ & $2 \%$ & $7 \%$ & $0.109(22)$ & $5.220(22)$ & $0.478(44)$ \\
\hline $\mathrm{SD}$ & $2 \%$ & $10 \%$ & $8 \%$ & $3 \%$ & $3 \%$ & $7 \%$ & $3 \%$ & $4 \%$ & $10 \%$ & 0.897 & 0.014 & 0.752 \\
\hline \multicolumn{13}{|l|}{$\%$ Goal } \\
\hline Mean & $24 \%$ & $35 \%$ & $39 \%$ & $37 \%$ & $46 \%$ & $53 \%$ & $45 \%$ & $38 \%$ & $43 \%$ & $6.049(22)$ & $1.898(22)$ & $1.472(44)$ \\
\hline $\mathrm{SD}$ & $16 \%$ & $24 \%$ & $24 \%$ & $24 \%$ & $20 \%$ & $25 \%$ & $25 \%$ & $18 \%$ & $27 \%$ & 0.008 & 0.174 & 0.227 \\
\hline \multicolumn{13}{|c|}{ \% External } \\
\hline Mean & $6 \%$ & $11 \%$ & $10 \%$ & $10 \%$ & $16 \%$ & $14 \%$ & $24 \%$ & $29 \%$ & $26 \%$ & $8.089(22)$ & $0.882(22)$ & $0.029(44)$ \\
\hline $\mathrm{SD}$ & $8 \%$ & $15 \%$ & $15 \%$ & $17 \%$ & $19 \%$ & $20 \%$ & $24 \%$ & $26 \%$ & $25 \%$ & 0.002 & 0.428 & 0.998 \\
\hline
\end{tabular}

Table 1 : Mean values and Standard Deviations for cycling speed, in-lane\%, TotFix\%, \%path, \%Travel, \%goal and \%external. F-value, (degrees of freedom) and significance are given for effect of lane Width, cycling Speed and Width^Speed

\subsection{Fixation location}

Overall, the Fix\% of the four fixation types was different $\left(\mathrm{F}_{3,9}=411.459 ; \mathrm{p}<0.001\right)$. Path $(41 \pm 30 \%)$ and Goal fixations $(40 \pm 23 \%)$ were the dominant types of fixations, followed by External fixations $(10 \pm 13 \%$; $\mathrm{p}<0.05)$ and Travel fixations $(3 \pm 6 \% ; \mathrm{p}<0.05)$. Both lane width $\left(\mathrm{F}_{8,38}=4.432 ; \mathrm{p}=0.001\right)$ and cycling speed $\left(\mathrm{F}_{8,38}=2.384 ; \mathrm{p}=0.034\right)$ had an effect on fixation location but no interaction between lane width and cycling speed was found $\left(\mathrm{F}_{16,126}=0.577 ; \mathrm{p}=0.896\right)$.

Percentage of gaze towards the Path was higher when cycling at Slow speed than at Preferred $(\mathrm{P}=0.040)$ and Fast speed $(\mathrm{p}=0.013)$. No difference in \%Path was found between Preferred and Fast cycling speed $(\mathrm{p}=0.288)$. Percentage of Travel gaze at Fast speed was significantly higher than at Slow speed $(\mathrm{p}=0.003)$, whereas no difference was found for Preferred speed as compared to Slow $(\mathrm{p}=0.208)$ or Fast speed $(\mathrm{p}=0.133)$. Percentage of Goal fixations was lower at Slow than at Fast speed but difference did not reach significance $(\mathrm{p}=0.084)$. The percentage Goal fixations at preferred speed was not significantly different than that at high $(\mathrm{p}=0.254)$ and at low speed $(\mathrm{p}=0.427)$. No effect of cycling speed was found for \%External fixations ( $\mathrm{p}>0.100)$.

Looking to the path (\% Path fix) increased as the lane was more narrow $(\mathrm{p}<0.050)$ while $\%$ Goal was lower on the Narrow lane than on the wider lanes $(\mathrm{p}<0.050)$. No significant difference in \% Goal was found between the Wide and the Middle lane $(\mathrm{p}=0.340)$. Percentage External fixations was higher on the Wide lane as compared to both the Middle $(\mathrm{P}=0.003)$ and Narrow lane $(\mathrm{p}=0.014)$. No difference in \%External was found between the Middle and the Narrow lane $(p=0.237)$ and no effect of lane width on \% Travel was found ( $p>0.100)$.

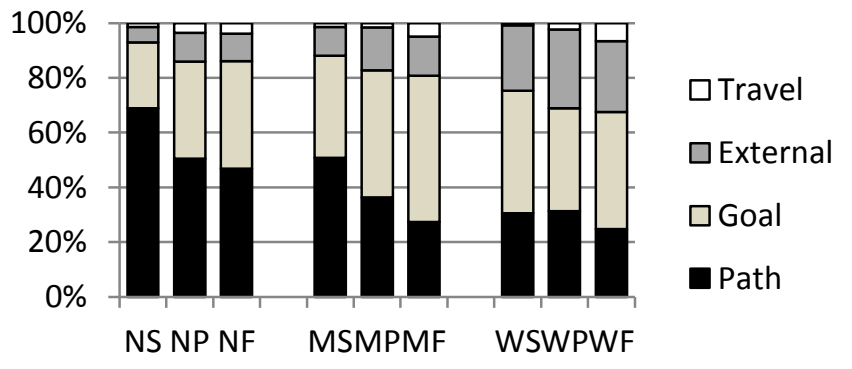

Figure 2 : Distribution of the four fixation types at different path widths (Narrow - Middle - Wide) and speeds (Slow Preferred - Fast). For values and significance, see text and Table 1 


\subsection{Travel Fixations versus OptoKinetic Nystagmus}

Only $3 \%$ of all fixations were categorized as travel fixations. Instead of travel fixations, OKN was revealed when participants watched the path. An example of how the OKN can be observed in the Y-coordinates of the gaze is given in Figure 3 (participant $X$ ), with each 'sawtooth' representing an OKR followed by a regressive saccade. If this participant would have made mainly travel fixations, the graph would show multiple horizontal lines. The Y-coordinates of participant $\mathrm{Y}$ show a typical 'goal-watching' behaviour.

\section{Discussion}

The overall aim of this paper was to investigate to what extent visual strategies documented for car drivers and pedestrians also hold for cycling. Our study results showed that participants mainly watched the path and the goal region but proportions were subject to lane width and cycling speed. Few travel fixations were measured, instead an Optokinetic Nystagmus was revealed.

Although not instructed to do so, participants cycled slightly faster when the cycling lane was wider. This effect of lane width on travelling speed has been previously described in car driving (McLean and Hoffman, 1972) and can be explained by the speedsteering workload trade-off. According to this concept, higher speeds and more narrow lanes both require a higher mental effort to keep the vehicle in lane. Therefore, speed will be adjusted according to the width of the cycling lane to keep the mental workload at a reasonable level for keeping the vehicle in position
(Godley et al., 2004). Adjustment of speed to the task difficulty is also called task difficulty homeostasis and is an essential part of the task-capability interface (TCI) model. According to this model driving behaviour can be described by the interaction between the determinants of task demand and the driver's capability (Ray Fuller, 2005; R. Fuller et al., 2008). The TCI-model was designed for car driving behaviour but from our data seems to be applicable for cycling behaviour as well.

Results of the gaze location analysis indicated a visual behaviour that was similar to that of pedestrians, with gaze directed to the path as well as to the goal area (Patla and Vickers, 2003; Turano et al., 2001; Foulsham et al., 2011). In contrast to the two-level model of steering during car driving, the near region was actively looked at. As Land and Horwood (1995) suggested, at lower speeds the near region seems to be sufficient for both stability and guidance. However, the high standard deviation of the fixation location suggests notable individual differences. In some trials, a visual behaviour resembling the two-level model of steering was revealed, characterized by prolonged fixations to the goal. In other trials gaze was directed primarily to the near path or even to external regions. It has been frequently shown that visual behaviour is highly task dependent (Yarbus, 1967; Marigold and Patla, 2007; Ballard and Hayhoe, 2010). The present results however suggest that large individual differences can also exist within the same task. In other words, multiple visual strategies can lead to the same visual information and an associated motor action. The observed variation of visual behaviour could be due to the specific task characteristics (i.e., self-imposed speed,

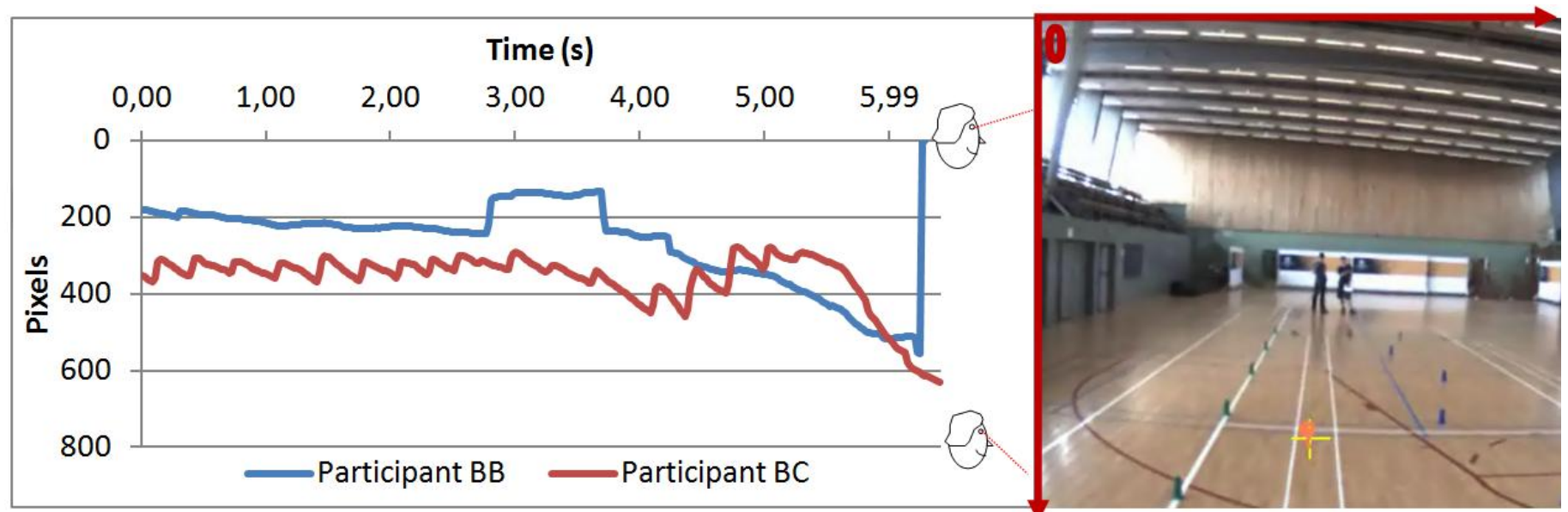

Figure 3 : Y coordinates of gaze of two participants while cycling on the $15 \mathrm{~m}$ track. Participant X looks at the path while OKN takes place and looks at the final gate about 1/2s before he reaches it ('path-watching'). Participant $Y$ looks at the final gate from the beginning, makes a shift towards an external region and then looks back at the final gate until he reaches it ('goal-watching'). 
lane width, etc.) or to a combination of individual differences such as cycling skills (balance and steering control (Ducheyne et al., 2012), perception of the task, visual dependency, etc.). An example of the visual behaviour during 'goal-watching' and 'path-watching' is shown in Figure 3. Notwithstanding the individual differences, participants shifted their attention towards the goal at higher speeds, towards the near pathway on narrow lanes and more towards irrelevant areas on wider lanes.

Since human action is associated with a visual-motor delay (Hayhoe and Ballard, 2005; Land, 2006), a visual buffer of 0,80 to 2,00 seconds is used in locomotion (Land and Furneaux, 1997; Wilkie and Wann, 2003; Wilkie et al., 2008). Considering a visual buffer of one second, participants in our current experiment should have looked approximately 2,5 meter in front of them in the slowest condition. In the preferred and high speed condition however, the same visual buffer will lead to look-ahead fixations at a distance of 3,3 and 4,7 meter respectively. With gaze often stabilized on the goal once it is within the range of the visual buffer, larger lookahead fixations will lead to a higher proportion of goal fixations. In other words, the higher proportions of goal fixations in the faster conditions are not necessarily the result of a different visual strategy but rather of the same visual buffer that got larger spatial dimensions as cycling speed was higher.

The effect of wider lane width on the visual behaviour could be explained by the lower task demand that it induces. It has been shown that selective attention to relevant stimuli is indicative for the information needs (Hughes and Cole, 1988; de Waard, 1996). So it is reasonable to suggest that less demanding situations will lead to a less restricted visual search pattern, and therefore to more task-irrelevant fixations. On the wide lanes participants can probably keep the bicycle on the track by using primarily the peripheral vision, analogous to the two-level model. But as the demands of traffic situations increase, the use of the peripheral vision will drop in favour of the information uptake by the fovea (Miura, 1987). So when the lane gets more narrow, peripheral vision is no longer sufficient for the lane keeping task and gaze will be directed closer to the bicycle. This shift of attention is similar to that during walking, where vision is also directed more to the path itself when the task becomes more demanding (Pelz and Rothkopf, 2007). Although in the study of Pelz and Rothkopf the higher task demand for walking is mainly due to a higher need for balance and that of cycling in the current study rather to a need for finer steering adjustments, both could be seen as a need for more direct control.

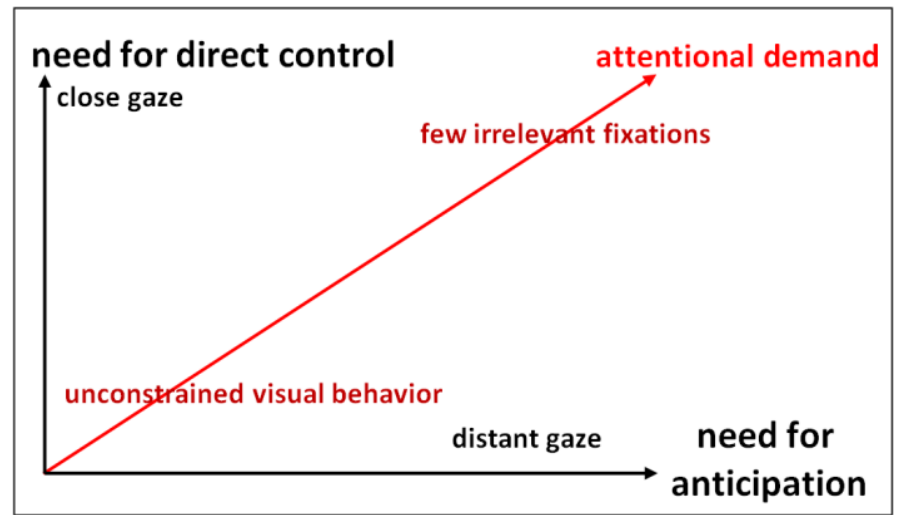

Figure 4 : Gaze constraints model for goal directed locomotion. The synergy of the need for direct control (task complexity, capability, ...) and the need for anticipation (unpredictability of environment, speed, ...) determines the gaze direction and attentional demand of the person in locomotion

A new gaze constraints model for goal directed locomotion is presented in Figure 4. Similar to the twolevel model of steering, it relies on the assumption that both the far region (guidance) and the near region (lanekeeping) are necessary for efficient steering. Different from the two-level model is that the current model is applicable to multiple forms of locomotion and predicts how gaze behaviour changes under different task and environmental constraints. The gaze constraints model assumes that reaching a goal in a safe manner requires: (1) direct control for stability and vehicle control and (2) anticipation for guidance and hazard perception. The need for direct control is characterised by close gaze behaviour (the 'near' region). This need increases with task complexity, and decreases with automatisation and/or mastery of the vehicle. The need for anticipation on the other hand is characterised by distant gaze behaviour (the 'far' region) and increases with speed as well as the extent to which the environment is unpredictable. Together with an increasing need for both direct control and anticipation, the attentional demand 
and mental workload will also increase, leaving less room for irrelevant gaze behaviour. Similar to the use of the two-level model (Salvucci and Gray, 2004), the near region will be attended peripherally and/or with intermittent fixations when the need for anticipation is higher than the need for direct control. When the need for direct control is highest, vision will be more similar to that of pedestrians on rough surface (Marigold and Patla, 2007), with gaze primarily directed to the near path with occasionally shifts to the distant regions.

The third aim of the present experiment was to find out whether travel fixations were used or an OptoKinetic Nystagmus (OKN) could be revealed. In contrast to previous findings of Patla and Vickers (2003) during walking, few travel fixations were observed during a cycling task. Alternatively, as was found for curve driving by Authié and Mestre, (2011) gaze towards the path directly in front of the participants was subject to an Optokinetic Nystagmus (OKN). To our knowledge the current paper is the first study to report the OKN in a non-simulated locomotion experiment. Results also disprove two of the suggestions made by Authié et al. (2011). They suggested that OKN may not have been observed in other studies due to an insufficient temporal resolution of the eye tracking systems being used as well as to the lower speeds of locomotion. In the current experiment, however, OKN was observed even though the temporal resolution of the eye tracker was $50 \mathrm{~Hz}$ and cycling speeds were between 6 and $26 \mathrm{~km} / \mathrm{h}$. Another possibility is that the methodology used for analysing eye movements determined whether or not OKN can be detected. Since eye movement data analysis is timeconsuming, many researchers rely on analysis software for fixation duration, location, etc. Unfortunately, this software usually does not detect smooth pursuit, which is an essential part of the OKN. Therefore, this particular visual behaviour might be missed out. This difference in methodology could also explain why few travel fixations were observed in the current study. If we look at the raw data of gaze (i.e., frame by frame eye movements) it seems as there is a 'travel gaze behaviour' in that way that gaze stays within a certain array in front of the body but keeps moving back and forth as a result of the OKN. If processed data (i.e., per fixation) of the analysis software are used, fixations keep reappearing on the same distance of the cyclist. Still, some travel fixations were observed, especially at higher speeds. These could be caused by a failing optokinetic reflex at higher travelling speeds or could be due to the gain ratio between gaze velocity and optical flow velocity being lower than one. OKR gain falls below unity at stimulus speeds exceeding 30\% (Howard and Ohmi, 1984). Similarly, Authié \& Mestre (2011) also reported gains of 0.66. A low gain of the OKR could have led to a visual behaviour resembling travel fixations. An alternative explanation is that these observations were the result of the frame by frame analysis. At higher travelling speeds, eye movements were somewhat more troubled and could have lead to a higher chance of falsely appointing a fixation as a travel fixation.

The presented study is a first step into understanding the visual behaviour of cyclists. However, current experiment only tested the model in an indoor, distraction-free environment, and visual behaviour was analyzed on a track of only fifteen meters long. This setup might have been too pragmatic to encounter some variations of in-traffic gaze behaviour. Therefore, further research is necessary to test if our proposed model is applicable in more open and realistic settings and if the OKN is also elicited in free cycling situations. Nevertheless, even on this short track, the effects of path width and cycling speed were apparent. Together with hazard perception and decision making, more insights in the visual behaviour of bicyclists could lead to primary prevention measures such as better infrastructure and adapted traffic education for children and adults.

\section{Conclusion}

Bicycle users adapted their cycling speed to the lane width in accordance to the speed-steering workload trade-off. Visual behaviour of the participants could only partly be explained by the two-level model. Participants shifted their attention towards the goal at higher speeds, towards the near pathway on narrow lanes and more towards irrelevant areas on wider lanes. Based on these findings, a gaze constraints model for goal directed locomotion was presented. Finally, few travel fixations 
were found, instead an optokinetic nystagmus was revealed.

\section{Acknowledgements}

This research was supported by the life line campaign of the Research Foundation of Flanders (FWO) FWO G.A115.11N. We also thank Stijn Van Goylen, Linus Zeuwts, Dennis Dieussaert and their colleagues for data collection and Davy Spiessens and Joeri Gerlo for the technical support.

\section{References}

Authié, C.N., Mestre, D.R., 2011. Optokinetic nystagmus is elicited by curvilinear optic flow during high speed curve driving. Vision research, 51 (16), 1791-800.

Ballard, D.H., Hayhoe, M.M., 2010. Modelling the role of task in the control of gaze. Visual Cognition, 17, 1185-1204.

Donges, E., 1978. A Two-Level Model of Driver Steering Behavior. Human Factors, 20 (6), 691707.

Ducheyne, F., De Bourdeaudhuij, I., Lenoir, M., Spittaels, H., Cardon, G., 2012. Children's cycling skills: Development of a test and determination of individual and environmental correlates. Accident Analysis and Prevention, In Press

Duchowski, A.T., 2007. Eye Tracking Methodology: Theory and Practice 2nd ed., London: springer.

Falkmer, T., Gregersen, N., 2005. A Comparison of Eye Movement Behavior of Inexperienced and Experienced Drivers in Real. Optometry and vision science, 82 (8), 732-739.

Foulsham, T., Walker, E., Kingstone, A., 2011. The where, what and when of gaze allocation in the lab and the natural environment. Vision research, 51 (17), 1920-31.

Fowler, G. A., Sherk, H., 2003. Gaze during visuallyguided locomotion in cats. Behavioural brain research, 139, 83-96.

Fuller, R., McHugh, C., Pender, S., 2008. Task difficulty and risk in the determination of driver behaviour.
European Review of Applied Psychology, 58 (1), 13-21.

Fuller, R., 2005. Towards a general theory of driver behaviour. Accident analysis and prevention, 37 (3), 461-72.

Godley, S.T., Triggs, T.J., Fildes, B.N., 2004. Perceptual lane width, wide perceptual road centre markings and driving speeds. Ergonomics, 47 (3), 237-256.

Hayhoe, M.M., Ballard, D., 2005. Eye movements in natural behavior. Trends in cognitive sciences, 9 (4), 188-94.

Hildreth, E.C., Beusmans, J.M., Boer, E.R., Royden, C.S., 2000. From vision to action: experiments and models of steering control during driving. Journal of experimental psychology. Human perception and performance, 26 (3), 1106-32.

Hollands, M.A., Patla, A.E., Vickers, J.N., 2002. "Look where you're going!": gaze behaviour associated with maintaining and changing the direction of locomotion. Experimental brain research. 143, 221230.

Howard, I.P., Ohmi, M., 1984. The efficiency of the central and peripheral retina in driving human optokinetic nystagmus. Vision research, 24 (9), 969-976.

Hughes, P.K., Cole, B.L., 1988. The Effect of Attentional Demand in Eye Movement Behaviour when Driving. In A. G. Gale et al., eds. vision in vehicles II. Proceedings of the second international conference on vision in vehicles. Nottingham, pp. 221-230.

Juhra, C., Wieskötter, B., Chu, K., Trost, L., Weiss, U., Messerschmidt, M., Malczyk, A., Heckwolf, M., Raschke, M., 2012. Bicycle accidents - Do we only see the tip of the iceberg? A prospective multicentre study in a large German city combining medical and police data. Injury, 43, 2036-2034

Kandil, F.I., Rotter, A., Lappe, M., 2009. Driving is smoother and more stable when using the tangent point. Journal of Vision, 9 (1), 1-11.

Knapp, C.M., Gottlob, I., McLean, R.J., Proudlock, F.A., 2008. Horizontal and vertical look and stare optokinetic nystagmus symmetry in healthy adult 
volunteers. Investigative ophthalmology \& visual science, 49 (2), 581-8.

Land, M.F., 2006. Eye movements and the control of actions in everyday life. Progress in retinal and eye research, 25, 296-324.

Land, M.F., Furneaux, S, 1997. The knowledge base of the oculomotor system. Philosophical Transactions of the Royal Society, 352, 1231-1239.

Land, M.F., Horwood, J., 1995. Which parts of the road guide steering? Nature, 377 (6547), 339-40.

Land, M.F., Lee, D.N., 1994. Where we look when we steer. Nature, 369, 742-744

Lappe, M., Hoffman, K.-P., 2000. Optic flow and eye movements. International review of neurobiology, 44, 29-47.

Lappe, M., Pekel, M., Hoffman, K.-P., 1998. Optokinetic eye movements elicited by radial optic flow in the macaque monkey. Journal of neurophysiology, 79 (3), 1461-80.

Marigold, D.S., Weerdesteyn, V., Patla, A.E., Duysens, J., 2007. Keep looking ahead? Re-direction of visual fixation does not always occur during an unpredictable obstacle avoidance task.

Experimental brain research, 176 (1), 32-42.

Marigold, D.S., Patla, A.E., 2007. Gaze fixation patterns for negotiating complex ground terrain.

Neuroscience, 144 (1), 302-13.

Mars, F., 2008. Driving around bends with manipulated eye-steering coordination. Journal of Vision, 8 (11), $1-11$.

McLean, R.J., Hoffman, E.R., 1972. The effects of lane width on River Driving steering control and performance. In Proceedings of the Sixth Australian Road Research Board Conference. pp. 418-440.

Miles, F.A., 1998. The neural processing of 3-D visual information : evidence from eye movements. European Journal of Neuroscience, 10 (3), 811822.

Miura, T., 1987. Behavior oriented vision: functional field of view and processing resources. In J. K. O’Regan \& A. Lévy-Schoen, eds. Eye Movements: From Physiology to cognition. Amsterdam, pp. 563-572.
Mourant, R.R., Rockwell, T.H., 1972. Strategies of Visual Search by Novice and Experienced Drivers. Human Factors, 14 (4), 325-335.

Niemann, T., Lappe, M., Büscher, A., Hoffmann, K.P., 1999. Ocular responses to radial optic flow and single accelerated targets in humans. Vision research, 39 (7), 1359-71.

Patla, A.E., Greig, M., 2006. Any way you look at it, successful obstacle negotiation needs visually guided on-line foot placement regulation during the approach phase. Neuroscience letters, 397 (1-2), 110-114.

Patla, A.E., Vickers, J.N., 2003. How far ahead do we look when required to step on specific locations in the travel path during locomotion? Experimental brain research, 148 (1), 133-138.

Patla, A.E., Vickers, J.N., 1997. Where and when do we look as we approach and step over an obstacle in the travel path ? Neurophysiology, 8 (17), 36613665.

Pelz, J.B., Rothkopf, C., 2007. Oculomotor behavior in natural and man-made environments. In R. van Gompel et al., eds. Eye Movements: a window on Mind and Brain. Amsterdam: Elsevier, pp. 661676.

Rabl, A., de Nazelle, A., 2012. Benefits of shift from car to active transport. Transport Policy, 19 (1), 121131.

Salvucci, D.D., Gray, R., 2004. A two-point visual control model of steering. Perception, 33 (10), 1233-1248.

Solomon, D. Cohen, B., 1992. Stabilization of Gaze During Circular Locomotion in Light I .

Compensatory Head and Eye Nystagmus in the Running Monkey. Journal of neurophysiology, 67 (5), 1146-1157.

Turano, K.A., Geruschat, D.R., Baker, R.H., Stahl, J.W., Shapiro, M.D., 2001. Direction of gaze while walking a simple route: persons with normal vision and persons with retinitis pigmentosa. Optometry and vision science, 78 (9), 667-75.

Turano, K.A., Geruschat, D.R., Baker, F.H., 2003. Oculomotor strategies for the direction of gaze 
tested with a real-world activity. Vision research, 43 (3), 333-46.

Underwood, G., Chapman, P., Brocklehurst, N., Underwood, J., Crundall, D., 2003. Visual attention while driving: Sequences of eye fixations made by experienced and novice drivers. Ergonomics, 46, 629-646.

de Waard, D., 1996. The Measurement of Drivers ' Mental Workload. University of Groningen.
Wilkie, R.M., Wann, J.P., 2003. Eye-movements aid the control of locomotion. Journal of Vision, 3, 677684.

Wilkie, R.M., Wann, J.P., Allison, R.S., 2008. Active gaze, visual look-ahead, and locomotor control. Journal of experimental psychology. Human perception and performance, 34 (5), 1150-1164. Yarbus, A.L., 1967. Eye Movements and Vision, New York: Plenum Press. 